\title{
Non-Bacterial Thrombotic Endocarditis: A Manifestation of Lung Cancer
}

\author{
Al Chalaby S, Aman E, Katiby Y, Vipparla NS, Mahdi AA, Amsterdam EA and Venugopal S* \\ Division of Cardiovascular Medicine, UC Davis School of Medicine, University of California, USA
}

"Correspondence: Sandhya Venugopal, Division of Cardiovascular Medicine, UC Davis School of Medicine, University of California, USA

Received on 14 November 2019; Accepted on 10 January 2020; Published on 14 January 2020

Copyright (C) $2020 \mathrm{Al}$ Chalaby S, et al. This is an open access article and is distributed under the Creative Commons Attribution License, which permits unrestricted use, distribution, and reproduction in any medium, provided the original work is properly cited.

Keywords: non-bacterial thrombotic endocarditis, lung cancer

\begin{abstract}
Abbreviations: NBTE: non-bacterial thrombotic endocarditis; DVT: deep vein thrombosis; CT: computed tomography; TTE: transthoracic echocardiogram; TEE: transesophageal echocardiogram; IE: infective endocarditis; MRI: magnetic resonance imaging
\end{abstract}

\section{Presentation}

Non-bacterial thrombotic endocarditis (NBTE) is characterized by vegetations on cardiac valves consisting of fibrin and platelet aggregates that are devoid of inflammation or bacteria. Diagnosis is often challenging and is aided by a high index of suspicion. These factors are reflected by the case of a 53 year old woman with a history of recurrent idiopathic deep vein thrombosis (DVT) prior to presentation at our institution to which she was admitted because of right upper extremity numbness and weakness.

\section{Assessment}

The patient's pertinent examination showed unremarkable vital signs; undisplaced point of maximum cardiac impulse, regular cardiac rhythm, normal heart sounds with no murmurs or gallops; clear lungs and a soft nontender abdomen. Neurological examination identified reduced strength in the right upper extremity: right wrist flexion and extension revealed moderately reduced strength and right-sided pronator drift. There was no evidence of splinter or conjunctival hemorrhages, Janeway lesions, Osler nodes, or Roth spots.

\section{Diagnosis}

The patient's laboratory results were remarkable for mild normocytic anemia (hemoglobin $11.1 \mathrm{~g} / \mathrm{dL}$; ref $12-15.5$ $\mathrm{g} / \mathrm{dL}$ ), thrombocytopenia 91,000 per $\mathrm{mm}^{3}$ (ref 150,000-450,000 per $\mathrm{mm}^{3}$ ) and troponin I $3.29 \mathrm{ng} / \mathrm{mL}$ (ref $<0.05$ $\mathrm{ng} / \mathrm{mL}$ ). The second troponin $6 \mathrm{~h}$ after presentation was $2.64 \mathrm{ng} / \mathrm{mL}$. Electrocardiogram revealed sinus bradycardia, normal axis, and no ST/T changes suggestive of ischemia (Figure 1). Computed tomography (CT) of the head was negative for intracranial hemorrhage, mass, or ischemia. Lower extremity duplex ultrasound was negative for DVT.

Magnetic resonance imaging (MRI)/magnetic resonance angiography (MRA) of the brain revealed multiple small and medium-sized infarcts whose distribution was consistent with embolic origin (Figure 2). 




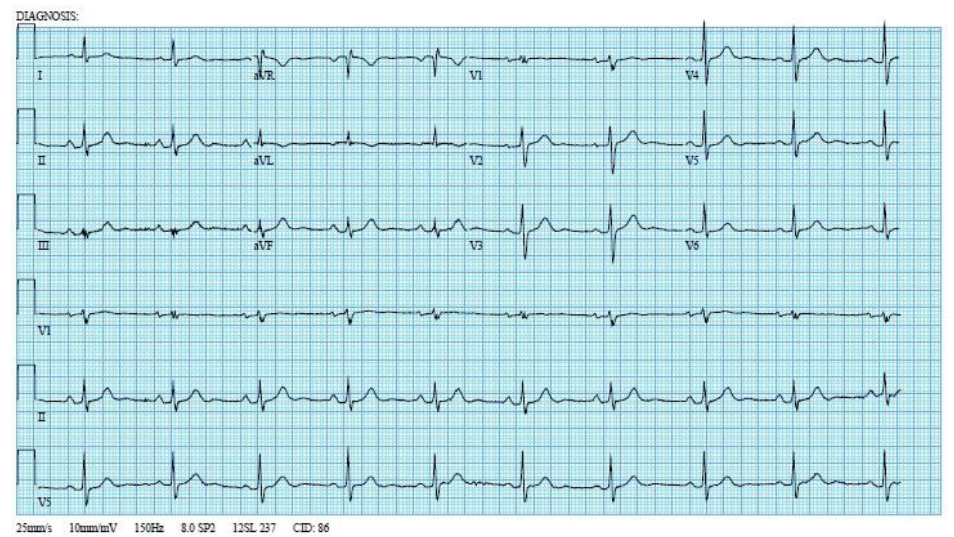

Figure 1: Electrocardiogram with sinus bradycardia.



Figure 2: Brain magnetic resonance imaging/magnetic resonance angiography with evidence of infarcts in right and left cerebellar hemispheres (outlined). L: left; R: right.

Transthoracic echocardiogram (TTE) was unremarkable, including no evidence of patent foramen ovale. Transesophageal echocardiogram (TEE) identified small irregular masses on the aortic valve consistent with vegetations (Figure 3). Serial blood cultures were negative.

Embolic phenomena in the absence of bacteremia raised the question of occult malignancy or autoimmune disease. The tests for anti-nuclear antibody, anti-neutrophil cytoplasmic antibody, antiphospholipid antibodies, and factor V Leiden mutation were negative. Levels of homocysteine, protein S, protein C, and antithrombin III were normal. However, the patient's chest CT revealed a $3.4 \mathrm{~cm}$ right lower lobe mass suggestive of malignancy and bronchoscopy guided-biopsy confirmed adenocarcinoma by histopathology (Figure 4). Abdominal and pelvic CT scans identified multiple wedge-shaped splenic and renal infarcts consistent with widespread embolization. The patient was diagnosed with NBTE in the setting of lung adenocarcinoma complicated by systemic thromboembolism. Treatment was initiated with intravenous unfractionated heparin followed by low molecular weight heparin and the patient was transferred to the oncology service for further management. 


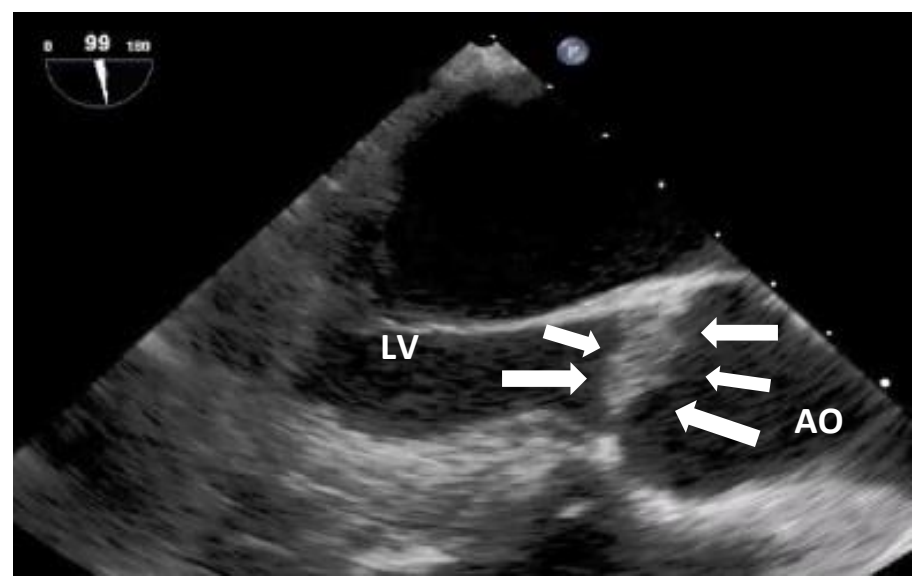

Figure 3: Transesophageal echocardiography revealing small, irregular vegetations on the aortic valve (arrows). LV: left ventricle; AO: ascending aorta.

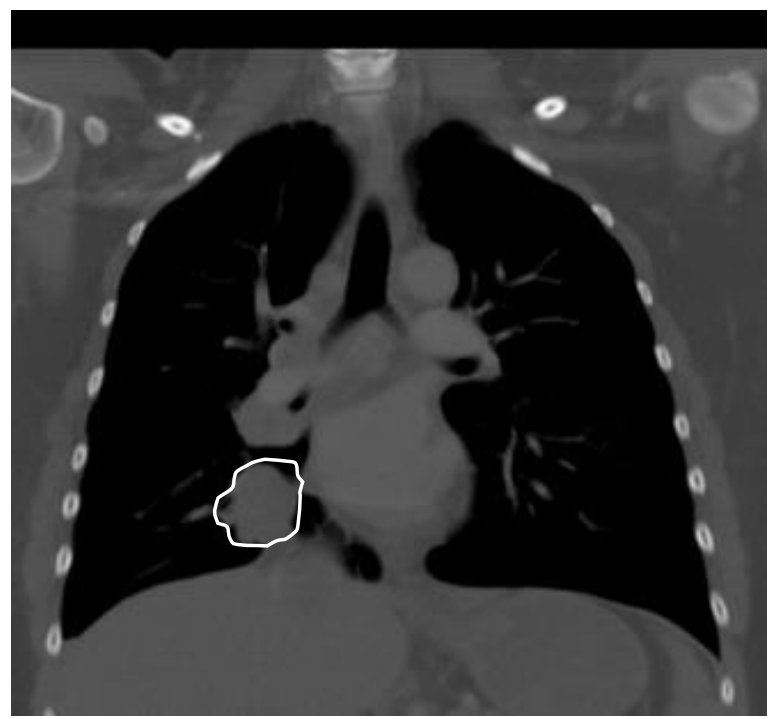

Figure 4: Coronal view of chest computed tomography demonstrating a $3.4 \mathrm{~cm}$ right lower lobe mass proven to be malignant (outlined).

NBTE depicts a spectrum of sterile lesions ranging from microscopic aggregates of platelets to large vegetations on previously undamaged heart valves in the absence of bacteremia. The most commonly affected valves are the aortic and the mitral [1]. NBTE was previously referred to as marantic endocarditis; "marantic" stems from the Greek word marantikos for "wasting away", signifying the seriousness of conditions associated with NBTE. The ante-mortem diagnosis of NBTE is made from the triad of systemic embolization, multiple valvular vegetations and the presence of a systemic disease known to be associated with NBTE. In comparison, diagnosis of infective endocarditis (IE) is confirmed by clinical evidence as provided by the Duke criteria in the setting of pathologically verified bacteremia [2]. Echocardiography is mandatory in all patients suspected of having endocarditis. Compared to TTE, TEE has significantly enhanced the diagnostic sensitivity for vegetative valvular lesions, perivalvular infection and mural abscess with a sensitivity of $80-90 \%[3,4]$.

Systemic detection of rheumatoid factor and antinuclear antibodies is recommended for all patients with suspected NBTE in association with malignancy or autoimmune disease [5]. Systemic embolization, as in our patient, occurs in nearly 50\% of patients with NBTE. The diagnosis of NBTE should be considered in any patient with a stroke and suspected malignancy. MRI patterns can help differentiate IE from NBTE [6,7]. One study of 36 patients with endocarditis revealed that 9 patients with NBTE had an MRI pattern of multiple small, medium or large disseminated lesions. The remaining 27 patients with IE had differing radiological patterns from NBTE; they either had a single lesion, a territorial infarction, or multiple punctate disseminated lesions [6]. 
Importantly, clinical evaluation of patients with endocarditis should be complete, including a detailed history and thorough examination that includes a pelvic assessment, standard blood tests, and a chest roentgenogram [8]. Advanced stages of malignancy, either solid or hematological, have been documented in patients with NBTE [8]. Similarly, recurrent idiopathic DVT should also be carefully evaluated for an underlying systemic condition. The triad of mucinous malignancies, venous thrombosis, and terminal endocarditis with emboli has been documented, signifying the necessity to search for other components of the triad when one is encountered [9].

\section{Management}

In general, treatment of NBTE is directed toward the underlying cause, in this case pulmonary malignancy. Systemic anticoagulation is also started given the risk of recurrent thromboembolism. Because NBTE has been considered part of the spectrum of Trousseau's syndrome, long-term use of heparin has been recommended and has prevented further development of cardiac valvular NBTE vegetations [5]. Postmortem findings have demonstrated that therapy with heparin also reduces the risk of cerebral infarction in patients with NBTE associated with malignancy [10]. A recent study in patients with malignancy and acute thromboembolism demonstrated low-molecular-weight heparin to be more effective than warfarin in reducing the risk of recurrent thromboembolism in NBTE [11-14]. Furthermore, anticoagulation should be continued indefinitely in patients with NBTE because of the risk of recurrent thromboembolism following discontinuation of this therapy.

\section{Conclusion}

Judicious evaluation of our patient's recurrent DVT by chest radiographic imaging and utilization of prophylactic systemic anticoagulation could have helped in the early diagnosis of her underlying malignancy and potentially prevented the devastating sequelae of systemic embolization and ischemic stroke.

\section{Author Contributions}

All authors had access to the data and participated in writing the manuscript.

\section{Conflicts of Interest}

All authors declare that we have no conflicts of interest.

\section{References}

1. Grimaldi A, De Gannero L, Vermi AC, et al. Cardiac valve involvement in systemic siseases: a review. Clin Cardiol. 2013;36(3):117-24.

2. Singhal AB, Topcuoglu MA, Buonanno FS. Acute ischemic stroke patterns in infective and nonbacterial thrombotic endocarditis: a diffusion-weighted magnetic imaging study. Stroke. 2002;33(5):1267-73.

3. Shapiro SM, Young E, De Guzman S, et al. Transesophageal echocardiography in diagnosis of infective endocarditis. Chest. 1994;105(2):377-82.

4. Sekar P, Johnson JR, Thurn JR, et al. Comparative sensitivity of transthoracic and transesophageal echocardiography in diagnosis of infective endocarditis among veterans with staphylococcus aureus bacteremia. Open Forum Infect Dis. 2017;4(2):1-8.

5. Katsouli A, Massad MG. Current issues in the diagnosis and management of blood culture-negative infective and non-infective endocarditis. Ann Thorac Surg. 2013;95(4):1467-74.

6. Asopa S, Patel A, Khan OA, et al. Non-bacterial thrombotic endocarditis. Eur J Cardiothorac Surg. 2007;32(5):696-701. 
7. Elagha A, Mohsen A. Cardiac MRI clinches diagnosis of Libman-Sacks endocarditis. Lancet. 2019;393(10182): 30770-6.

8. Hettiarachchi RJ, Lok J, Prins MH, et al. Undiagnosed malignancy in patients with deep vein thrombosis: incidence, risk indicators, and diagnosis. Cancer. 1998;83(1):180-85.

9. Rohner RF, Prior JT, Sipple JH. Mucinous malignancies, venous thrombosis and terminal endocarditis with emboli: A syndrome. Cancer. 1966;19(12):1805-12.

10. Rogers LR, Cho ES, Kempin S, et al. Cerebral infarction from non-bacterial thrombotic endocarditis: Clinical and pathological study including the effects of anticoagulation. Am J Med. 1987;83(4):746-56.

11. Lee AY, Levine MN, Baker RI, et al. Low-Molecular-Weight Heparin versus a Coumarin for the Prevention of Recurrent Venous Thromboembolism in Patients with Cancer. N Engl J Med. 2003;349(2):146-53.

12. Arnautovic JZ, Yamasaki H, Rosman HS. Multiple embolic strokes as a result of Libman-Sacks endocarditis associated with lupus and secondary antiphospholipid antibody syndrome: a case report. Eur Heart J Case Rep. 2018;2(3):1-6.

13. Bazyar Z, Moaref A, Amirghofran AA, et al. A woman with systemic lupus erythematosus and odd valvular presentation: a case report. Am J Case Rep. 2019;20:1705-08.

14. Zaman S, Rahim MA, Sayami LA, et al. Libman-sacks endocarditis in a Bangladeshi patient suffering from rhupus. Trop Doct. 2019;49(4):309-11. 